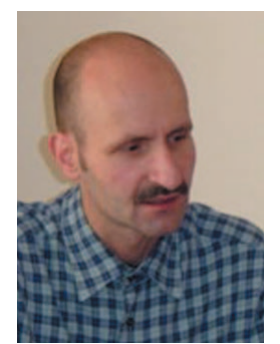

Gerd Dietrich

\section{Psychoedukation in der Behandlung bipolarer affektiver Erkrankungen}

\author{
Gerd Dietrich, Petra Wagner, Peter Bräunig
}

Klinikum Chemnitz gGmbH, Klinik für Psychiatrie, Verhaltensmedizin und Psychosomatik, Chemnitz

psychoneuro 2003; 29 (9): 403-408
I n der Behandlung bipolarer affektiver Erkrankungen dominieren noch immer pharmakologische Behandlungsstrategien. Auf der Basis einer hypothetisierten Ätiopathogenese, für die biologische Determinanten entscheidend sind, wurde das Auftreten von Rezidiven ursächlich in erster Linie auf medikamentöse Noncompliance des Betroffenen zurückgeführt. Studienergebnisse zu Beginn der 90er-Jahre, die aufzeigten, dass trotz complianter Einnahme der rezidivprophylaktischen Medikation bei ca. $75 \%$ der Betroffenen im 5-Jahresintervall ein Rezidiv auftrat, führten zu einer Modifikation der Ätiologievorstellungen in Richtung eines adäquater erscheinenden biopsychosozialen Erkrankungsmodells. Dieses Modell trägt dabei vor allem der Beobachtung Rechnung, dass soziale bzw. interpersonelle Faktoren - als Stressoren wirkende Lebensereignisse bei der erneuten Exazerbation der Symptomatik eine wichtige Rolle spielen.

Die ursprüngliche Favorisierung biologischer Komponenten bei Krankheitsgenese und -verlauf, hatte zur Folge, dass relativ spät, eigentlich erst mit Integration der psychosozialen Betrachtungsweise, intensive Bemühungen gestartet

Basierend auf einem biologisch determinierten Ätiologiekonzept wurde über viele Jahre in der Behandlung bipolarer Erkrankungen die medikamentöse Therapie favorisiert. Empirische Befunde, die aufzeigten, dass trotz Einhaltung des medikamentösen Behandlungsregimes nahezu 75\% der Betroffenen im 5-Jahres-Intervall ein Rezidiv erleiden, führten zur Integration psychotherapeutischer Interventionsformen in die Behandlung bipolarer Erkrankungen. Adjuvant zur medikamentösen Therapie scheint Psychoedukation - die Informationsvermittlung über die Erkrankung und ihre Behandlungsmöglichkeiten - eine vielversprechende Methode, den Krankheitsverlauf sowie die Krankheitsbewältigung günstig zu beeinflussen und insbesondere die medikamentöse Behandlungscompliance zu erhöhen.

wurden, psychotherapeutische Behandlungsverfahren für die bipolare Erkrankung zu entwickeln. Vielversprechend erscheinen dabei die Familienfokussierte Therapie (7), die Interpersonelle und Soziale Rhythmustherapie (6), kognitiv-verhaltenstherapeutische Verfahren $(1,9)$ sowie vorwiegend psychoedukativ orientierte Ansätze (16).

Alle Interventionsformen, so unterschiedlich sie auch sein mögen, basieren dabei mit unterschiedlicher Gewichtung auf Psychoedukation. In Abhängigkeit von der psychoedukativen Schwerpunktlegung fließen zusätzlich weitere Behandlungselemente mit ein, die sich an den von den jeweiligen Autoren favorisierten Einflussfaktoren auf Krankheitsentstehung und -verlauf orientieren.

So ist in der Familienfokussierten Therapie (7) bipolarer Erkrankungen neben psychoedukativen Elementen das familiäre Interaktionsverhalten ein zentrales Therapieziel. Untersuchungen zufolge sehen die Autoren ein familiäres Klima, in dem stark affektbesetzte Ausdrucks- und Verhaltensweisen vorherrschen und wenig Verständnis im täglichen Miteinander entgegengebracht wird, mit einem ungünstigen Krankheits- und Behandlungsverlauf assoziiert. Die Interpersonelle und Soziale Rhythmustherapie (6) hingegen hypothetisiert die Implikation interpersoneller Konflikte sowie die Derhythmisierung biologischer Zyklen als krankheitsauslösend bzw. -aufrechterhaltend. Demgegenüber sehen kognitiv-verhaltenstherapeutische Ansätze (1, 9) dysfunktionale Kognitionen in enger Verbindung mit der erneuten Symptomexazerbation.

Bevor wir uns im Weiteren auf die psychoedukativen Aspekte die- 
ser Ansätze konzentrieren, muss jedoch betont werden, dass entsprechend des derzeitigen Forschungsstandes nach wie vor im Rahmen einer wirkungsvollen Therapie bipolarer Erkrankungen die medikamentöse Behandlung als unbedingt erforderlich anzusehen ist. Psychotherapeutische bzw. psychoedukative Interventionen können nur als zusätzliche, unterstützende Behandlungsmaßnahmen gelten.

\section{Psychoedukation: \\ Was ist das?}

Allgemein wird mit „Psychoedukation“ die Informationsvermittlung über die Erkrankung und ihre Behandlungsmöglichkeiten bezeichnet, wobei psychologische Betrachtungsweisen Berücksichtigung finden. Alternativ könnte „Psychoedukation" auch so definiert werden, dass es sich hierbei um eine Infor- mationsvermittlung über die Erkrankung handelt, die dazu dient, die psychische Befindlichkeit zu verbessern bzw. zu stabilisieren. Ziel ist es, Selbsthilfemöglichkeiten im Umgang mit der Erkrankung zu aktivieren, die Behandlungscompliance $\mathrm{zu}$ erhöhen und damit das Wiedererkrankungsrisiko abzusenken.

$\mathrm{Zu}$ beachten ist, dass unter dem Begriff „Psychoedukation“ ganz unterschiedliche Arten der Wissensvermittlung zusammengefasst werden. So zählen hierzu Informationsbroschüren, Bücher, Videobänder, Fernsehsendungen, Internetseiten bzw. -foren bis zu elaborierten Gruppenprogrammen, die von mindestens einem ausgebildeten Therapeuten (Arzt, Psychotherapeut oder Psychologe) durchgeführt werden.

Da von allen psychoedukativen Interventionsformen der therapeutengeleiteten Form in der Behand-

\section{Abb. 1 Psychoedukation vs. Standardversorgung}

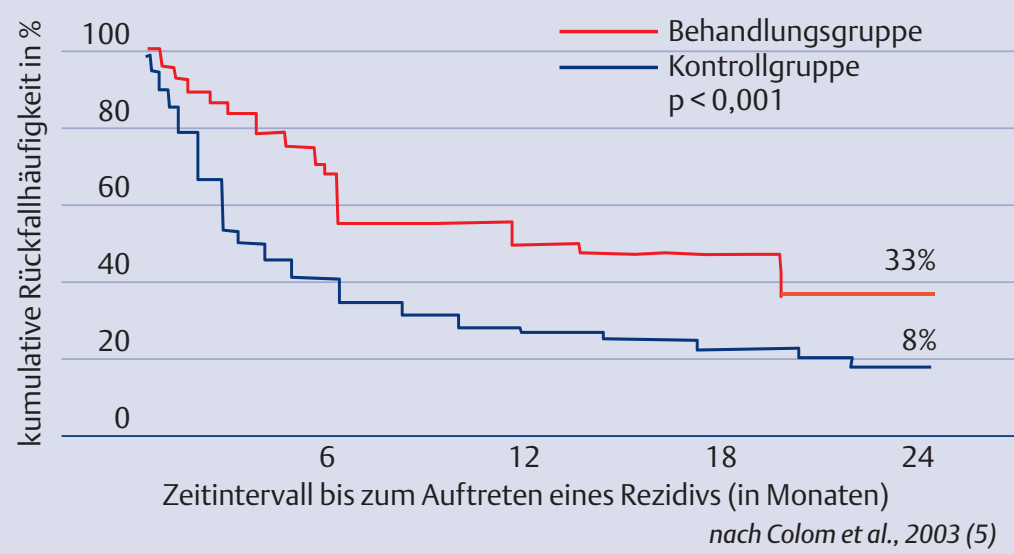

\section{Abb. 2 Psychoedukation vs. Standardversorgung}

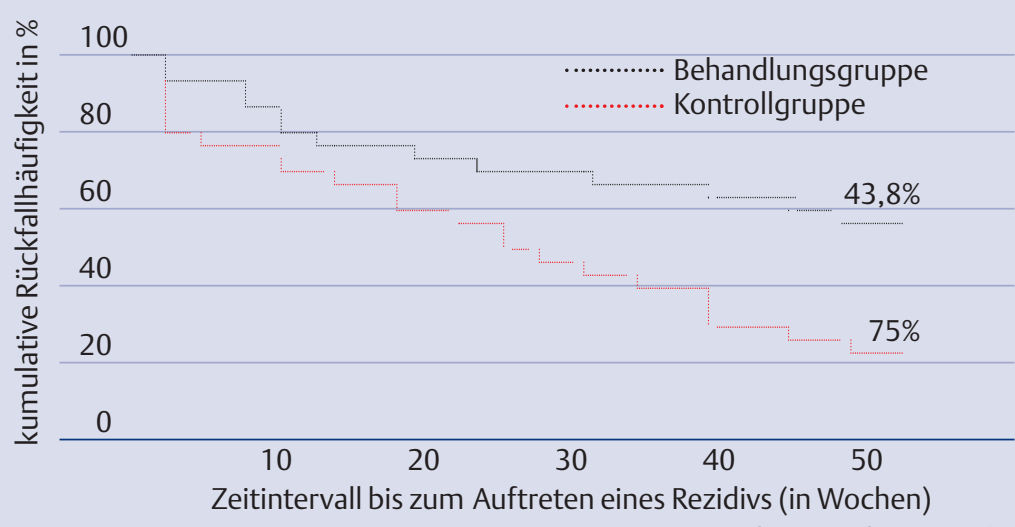

nach Lam et al., 2003 (10)

lung bipolarer Erkrankungen eine besondere Bedeutung zukommt, werden wir uns im Folgenden nur auf diese Variante der Psychoedukation beziehen.

\section{Entwicklung von psycho- edukativen Ansätzen in der Behandlung bipolarer Erkrankungen}

Die Idee der Psychoedukation ist nicht neu. Erste Ansätze wurden im Rahmen der Behandlung somatischer Erkrankungen, wie z.B. Diabetes oder kardio-vaskuläre Symptomatiken, entwickelt und konzentrierten sich hauptsächlich auf das Training der korrekten Einnahme von Medikamenten. Wegweisend waren hier Beobachtungen, die ungünstige Behandlungsverläufe in Zusammenhang mit der nicht ordnungsgemäßen Einnahme der Medikation sahen. Das so genannte Medikamententraining, d.h. die Einnahme der Medikation unter Aufsicht des Pflegepersonals, war zentraler Bestandteil der ersten Psychoedukationsformen.

Angewandt auf psychische Symptomatiken bewährte sich der psychoedukative Ansatz schon relativ frühzeitig in der Schizophreniebehandlung und gilt hier heute als grundlegendes therapeutisches Element.

Während erste psychotherapeutische Behandlungsversuche bipolarer Erkrankungen am familiären Unterstützungssystem anknüpften, wobei aus heutiger Sicht unklar bleibt, inwieweit hierbei psychoedukative Elemente eingebunden waren, wurde in der Folge gemäß der vorherrschenden biologisch orientierten Ätiologiehypothese in psychoedukativer Weise an der Erhöhung der medikamentösen Compliance gearbeitet. Praktisch sind daher erste psychoedukative Bemühungen in der Behandlung bipolarer Erkrankungen ebenfalls gleichbedeutend mit Medikamententraining (4). Neue Impulse in der Entwicklung psychotherapeutischer Interventionen resultierten dann $\mathrm{zu}$ Beginn der 90er-Jahre, basierend auf Befunden von Evaluationsstudien. Der neue psychotherapeutische Akzent bestand in einer Ausweitung 
der Perspektive hinsichtlich syndromauslösender bzw. -aufrechterhaltender Faktoren. Psychoedukation, ureigenstes Element kognitivverhaltenstherapeutischen Arbeitens, entstanden aus der praktischen Umsetzung des verhaltenstherapeutischen Axioms der Transparenz, erlangte inzwischen aufgrund ihres zeitökonomischen Charakteristikums und vielversprechender empirischer Evaluationsbemühungen eine Art Autonomie. Die oftmals getroffene Differenzierung zwischen Psychoedukation und Kognitiver Verhaltenstherapie bleibt allerdings aufgrund der Teil-Ganzes-Relation in gewisser Weise artifiziell, erscheint aber angesichts der Forderung moderner Forschungsstandards, nach kontrollierten empirischen Analysen therapeutischer Wirkfaktoren, hilfreich und sinnvoll. Festzuhalten bleibt, dass Psychoedukation im Zuge einer modernen Psychotherapieperspektive integrativer Bestandteil unterschiedlicher therapeutischer Richtungen ist. Das Ausmaß der Einbeziehung psychoedukativer Elemente variiert dabei, wie schon erwähnt, zwischen den therapeutischen Ansätzen.

\section{Rahmenbedingungen von Psychoedukation}

Therapeutengeleitete Psychoedukationsformen können nach Setting und Zielgruppe differenziert werden:

So gibt es zum einen Psychoedukation sowohl im stationären, tages- klinischen als auch ambulanten Rahmen. Zum anderen wird Psychoedukation in Abhängigkeit von der Teilnehmerzusammensetzung als Psychoedukation für Betroffene, für Angehörige oder für beide Teilnehmergruppen gemeinsam angeboten. Weiterhin kann unterschieden werden zwischen Psychoedukation in Einzel- oder Gruppenform.

Im Vergleich zur Psychoedukation im Einzelsetting scheinen Gruppenprogramme nicht nur aus ökonomischer Sicht einige Vorteile zu bieten.

Denn, auch wenn Gruppenprozesse bei psychoedukativen Gruppenprogrammen nicht primär im Vordergrund stehen, ist der Einfluss von Gruppenwirkfaktoren nicht zu unterschätzen und sollte unbedingt genutzt werden (17).

Denkt man insbesondere an krankheitsbedingtes Fehlverhalten, wie es während manischer Episoden auftritt, scheint doch die nur im Gruppensetting zu machende Erfahrung, anderen geht es genauso, den Betroffenen von der im Nachhinein oftmals schambesetzten Bewertung der Verhaltensexzesse enorm zu entlasten und damit zu helfen. Darüber hinaus sind die Möglichkeit der gegenseitigen Unterstützung und die Chance, aus den Erfahrungen anderer Betroffener zu lernen sowie generell das Gefühl, unter Mitbetroffenen eher verstanden zu werden, eindeutig positiv einzustufen.
Anzumerken ist, dass im Zuge der geforderten empirischen Evaluation von Psychoedukation als eigenständige Interventionsform in der Behandlung bipolarer Erkrankungen die Implikation von Gruppenwirkfaktoren allerdings konfundierend zu werten und gleichzeitig in entsprechenden Forschungsdesigns zu berücksichtigen ist.

\section{Inhalte psychoedukativer Ansätze}

Entsprechend den unterschiedlichen Bedürfnissen der Teilnehmergruppen unterliegen die Inhalte und Zielsetzungen geringfügigen Modifikationen. So muss in der psychoedukativen Arbeit mit Betroffenen der Umfang und die Art der Informationsvermittlung dem jeweiligen Erkrankungsstatus angepasst werden, um nicht Gefahr zu laufen, zu überfordern. Im Rahmen der Psychoedukation mit Angehörigen sollte der andere Blickwinkel auf die Erkrankung und ihre Folgen Berücksichtigung finden und damit der veränderten Bedürfnislage Rechnung getragen werden.

Aufgrund der im vorangegangenen Abschnitt angedeuteten Vorteile psychoedukativer Gruppenprogramme haben wir stellvertretend für alle psychoedukativen Ansätze deren Zielsetzungen in Tabelle 1 aufgeführt.

Übergeordnetes Ziel bleibt für alle Formen der Psychoedukation,

\section{Tab. 1 Ziele psychoedukativer Gruppenprogramme differenziert nach Teilnehmern}

\begin{tabular}{|c|c|c|}
\hline $\begin{array}{l}\text { Psychoedukative } \\
\text { Gruppen }\end{array}$ & Ziele & \\
\hline für Betroffene & $\begin{array}{l}\text { selbstverantwortlicher Umgang mit der Erkrankung } \\
\text { Aktivierung von Selbsthilfemöglichkeiten } \\
\text { über Erfahrungsaustausch Vermittlung des Gefühls, mit der Erkrankung } \\
\text { nicht allein zu sein, verstanden zu werden, Entlastung von Angst-, Schuld- } \\
\text { und Minderwertigkeitsgefühlen } \\
\text { Erhöhung der Behandlungscompliance }\end{array}$ & 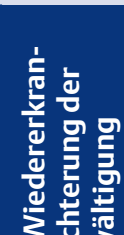 \\
\hline für Angehörige & $\begin{array}{l}\text { Schaffung von Verständnis für krankheitsbedingte Verhaltensweisen } \\
\text { Entlastung durch Erfahrungsaustausch } \\
\text { Angehörige/Bezugspersonen sozusagen zum „Behandlungsverbündeten“ } \\
\text { gewinnen, um hierdurch den Betroffenen im Umgang mit der Erkrankung } \\
\text { zu unterstützen }\end{array}$ & 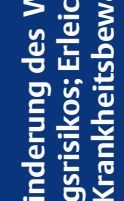 \\
\hline für Betroffene und Angehörige & $\begin{array}{l}\text { Schaffung wechselseitigen Verständnisses } \\
\text { Aktivieren der Familie/des sozialen Netzes als natürliche } \\
\text { Unterstützungsquelle }\end{array}$ & 量弪 \\
\hline
\end{tabular}


das Wiedererkrankungsrisiko abzusenken und den Umgang mit der Erkrankung zu erleichtern.

Hieraus ergeben sich für psychoedukative Behandlungsansätze folgende zentrale Themenschwerpunkte:

- Informationsvermittlung über die Erkrankung (Epidemiologie, Symptomatik, phänotypische Varianz, Ätiologie, Verlauf) und die Behandlungsmöglichkeiten

- Vermittlung von Copingstrategien, d.h. Hilfen, die den Umgang mit der Erkrankung erleichtern

- Rückfallprophylaxe.

Vergleichbar einem Baukastensystem werden in modernen Psychoedukationsprogrammen diese inhaltlichen Schwerpunkte in Form von sitzungsbezogenen „Themenbausteinen“ bzw. Modulen strukturiert und bearbeitet. Tabelle 2 gibt einen Überblick möglicher Module psychoedukativer Behandlungsprogramme. Die einzelnen Themenbausteine sollten dabei weniger in traditioneller Lehrermanier abgehandelt werden, als vielmehr gemäß moderner Unterrichtsdidaktik unter Einsatz ansprechender Materialien und technischer Hilfsmittel (Polylux, Flipchart) gemeinsam erarbeitet werden.
Unterstützung in der didaktischen Vorgehensweise erhalten Therapeuten durch entsprechende elaborierte Behandlungsmanuale, die sowohl für einzel- als auch gruppentherapeutische Maßnahmen vorliegen. Einen Überblick gibt hierzu Tabelle 3. Die Sitzungsfrequenz variiert zwischen 5-20 Stunden, die in der Regel im wöchentlichen Turnus stattfinden. Während einige Interventionsformen bereits in der akuten Phase einsetzen (6), scheint aus unserer Erfahrung die Indikation zur Psychoedukation erst in der subakuten Phase sinnvoll. In der Akutbehandlung erweisen sich aus unserer Einschätzung supportive Maßnahmen eher geeignet als psychoedukative.

Hinsichtlich Psychoedukation mit Angehörigen von bipolar Erkrankten liegt derzeit lediglich ein Programm in manualisierter Form vor (7). Sehr interessant, aber leider nicht manualisiert, erscheint diesbezüglich die Vorgehensweise von van Gent \& Zwart (15), deren fünf Sitzungen umfassendes Gruppenprogramm sich ausschließlich an Angehörige bipolar erkrankter Menschen richtet. Zentral sind neben der Vermittlung von Erkrankungswissen und Unterstützungsstrategien die Wahrnehmung eigener Bedürfnisse, die oftmals von Partnern psychisch erkrankter Menschen vernachlässigt wird. Letztgenannter Aspekt scheint dabei besonders wichtig, da hierdurch Frustrationen und Konflikte entstehen können, was sich wiederum ungünstig auf die Symptomatik des Betroffenen auswirken kann.

Abschließend ist darauf hinzuweisen, dass weitere Anregungen aus der Angehörigenarbeit im Rahmen der Schizophreniebehandlung entnommen werden können(3).

\section{Empirische Evaluation psychoedukativer Ansätze}

Generell basieren vergleichende Evaluationsstudien im Rahmen einer psychotherapeutischen Behandlung bipolarer Erkrankungen auf einer „Add-on-Strategie“, d. h. zusätzlich zur medizinischen Standardversorgung erfolgen psychoedukative Interventionen. Problematisch erscheint dabei, dass unter „Standardversorgung" einige Studienautoren eine vorwiegend medikamentöse Behandlung verstehen, andere wiederum niederfrequente therapeutische Kontakte mit einschließen, wodurch natürlich die Generalisierbarkeit der Ergebnisse sowie insgesamt die Erbringung eines Effektivitätsnachweises erschwert wird.

\section{Tab. 2 Synopsis von Modulen psychoedukativer Ansätze}

\begin{tabular}{|ll} 
Module & Kurzbeschreibung des Inhalts Modul \\
\hline Einführung & $\begin{array}{l}\text { Allgemeine Informationsvermittlung über die Erkrankung, ihre Epidemiologie, Ver- } \\
\text { laufscharakteristika und die Behandlungsmöglichkeiten }\end{array}$ \\
\hline Depression & $\begin{array}{l}\text { pathognomonische sowie individuelle Symptome, allgemeine bzw. individuelle Ver- } \\
\text { laufscharakteristika, typische dysfunktionale Kognitionen }\end{array}$ \\
\hline Manie & $\begin{array}{l}\text { pathognomonische sowie individuelle Symptome, allgemeine bzw. individuelle Ver- } \\
\text { laufscharakteristika, typische dysfunktionale Kognitionen }\end{array}$ \\
\hline Vermittlung eines Ätiologiemodellss & $\begin{array}{l}\text { Präferiert wird in den manualisierten Programmen die Erläuterung des Diathese- } \\
\text { Stress- bzw. Vulnerabilitäts-Stress-Modells, wobei potenzielle individuelle Ätiologie- } \\
\text { faktoren in das Modell integriert werden }\end{array}$ \\
\hline Medikamentöse Behandlung & $\begin{array}{l}\text { Detaillierte Information zur medikamentösen Behandlung; wichtige Fachbegriffe } \\
\text { werden erklärt. Insbesondere der Umgang mit Nebenwirkungen, die häufig Auslöser } \\
\text { medikamentöser Noncompliance sind, wird thematisiert }\end{array}$ \\
\hline Stressmanagement & Identifikation individueller Stressoren; Erlernen von Methoden zur Stressreduktion \\
& bzw. Stressbewältigung \\
\hline Rückfallprophylaxe & Erkennen individueller "Frühwarnzeichen"; Erlernen adäquater Reaktionsweisen auf \\
& Erstsymptome, z.B.: \\
& - bei Depression: Tagesstrukturierung, Planung positiv erlebter Aktivitäten \\
& - bei Manie: Aktivitätenreduktion, Stabilisierung des Schlaf-Wach-Rhythmus \\
& - episodenübergreifend: Symptomchecklisten; Führen von Stimmungsprotokollen; \\
& Erstellen eines Krisenplanes
\end{tabular}


Die ersten empirischen Evaluationsbemühungen blieben in der Regel auf Prä-Post-Vergleiche, teilweise ohne Einbeziehung einer adäquaten Kontrollgruppe, reduziert. Selbst das Gros der Experimentalgruppen war hinsichtlich der applizierten Medikation oder des Status der Symptomatik äußerst heterogen, oftmals nicht nach Erkrankungssubtypen spezifiziert; die Stichprobenwahl auf anfallende Stichproben von geringer Größe begrenzt.

Während somit Effektivitätsuntersuchungen in Form von prospektiven randomisierten Studien, die angemessene Katamnesezeiträume einschließen, in der Vergangenheit die große Ausnahme bildeten, um nicht zu sagen, fehlten, ist neuerdings ein Trend erkennbar, psychotherapeutische Interventionen in der Behandlung bipolarer Erkrankungen, und hier vor allem psychoedukative Ansätze im Rahmen kognitiv-verhaltenstherapeutischen Arbeitens, den modernen Forschungsstandards zu unterziehen (5, 10). Als Erfolgskriterien werden dabei hauptsächlich das Zeitintervall bis zur Wiedererkrankung, die medikamentöse Compliance, operationalisiert über Laborparameter, der Verlauf der Symptomatik, erfasst insbesondere über Selbstratings, die Hospitalisierungsrate bzw. -dauer und das psychosoziale Funktionsniveau gewählt.

Für psychoedukative Ansätze im Rahmen therapeutischen Arbeitens mit Betroffenen werden dabei in erster Linie günstige Ergebnisse berichtet. So wird für Teilnehmer an psychoedukativen Programmen eine geringere Rückfallrate $(5,10,14)$, ein längeres Zeitintervall bis zum Auftreten eines Rezidivs (5, 10; s. Abb. 1 u. Abb. 2), eine geringere Rehospitalisierungsrate $(4,5,10,14)$ sowie eine geringere Hospitalsisierungsdauer (5) festgestellt. Auch bezüglich des Erkennens und Einleitens geeigneter Maßnahmen im Zusammenhang mit Prodromen scheinen Betroffene von bipolaren Erkrankungen durch Informationsvermittlung zu profitieren (10). Darüber hinaus wird bei Psychoedukationsteilnehmern eine bessere medika- mentöse Compliance $(4,10)$ und ein höheres psychosoziales Funktionsniveau $(12,13,14)$ gefunden. Hinsichtlich der allgemeinen Akzeptanz psychoedukativer Maßnahmen werden ebenfalls positive Ergebnisse berichtet.

Allerdings werden vereinzelt auch weniger eindeutige Effekte ausgemacht (11), insbesondere auf subsyndromaler Störungsebene (10).

Die empirische Befundlage zu psychoedukativen Ansätzen, die Angehörige einbeziehen bzw. sich speziell an Angehörige richten, zeigt ähnlich günstige Untersuchungsergebnisse. Generell wird Psychoedukation von Angehörigen sehr positiv bewertet, Verständnisgewinn für den Betroffenen und Möglichkeit zum Erfahrungsaustausch werden dabei besonders hervorgehoben (15). Eine geringere Rückfallrate, ein längeres symptomfreies Intervall bis zum erneuten Auftreten eines Rezidivs (8), Verbesserungen hinsichtlich des psychosozialen Funktionsniveaus und der familiären Interaktion werden aufgefunden. Aber auch weniger eindeutige Effekte hinsichtlich diverser Krankheitsverlaufsparameter werden verortet, wobei das Informieren der Angehörigen über die Erkrankung bei Betroffenen zunächst auch Ängste auslösen kann (15).

\section{Fazit}

Psychoedukation adjuvant zur medikamentösen Behandlung bipolarer Erkrankungen scheint mit deutlich günstigeren Effekten hinsichtlich Behandlungscompliance, Symptommanagement, der allge- meinen Befindlichkeit und Leistungsfähigkeit assoziiert zu sein als eine rein pharmakologisch orientierte Behandlung der Erkrankung.

Der Indikationsbereich, welche Variante (Einzel, Gruppen, Einbeziehung von Angehörigen) für welchen Patienten zu welchem Zeitpunkt geeignet erscheint, bedarf noch weiterer empirischer Untersuchung. Interessante Konzepte, wie „extend of expressed emotions" (7) könnten dabei als wichtige Kriterien fungieren.

Leider liegen im Rahmen der Evaluation psychoedukativer Ansätze Prozess-Outcome-Analysen, die z.B. das Ausmaß des Erkrankungswissens oder die Implikation von Zuwendungs- oder Gruppeneffekten kontrollieren, noch nicht vor. Langzeitkatamnesen fehlen bislang. Auch erscheint zum gegenwärtigen Zeitpunkt die definitorische Expansion und damit die Subsumierung noch so unterschiedlicher psychotherapeutischer Interventionsformen mit mehr oder weniger elaborierten psychoedukativen Behandlungselementen unter den Psychoedukationsbegriff ungünstig. Die notwendige Systematisierung von Evaluationsversuchen sowie die Generalisierbarkeit der Ergebnisse werden hierdurch unnötig erschwert.

Forschungsbedarf besteht ferner hinsichtlich der Wirkprinzipien von Psychoedukation. Generell wäre denkbar, dass Zuwachs des Erkrankungswissens nicht nur mit einer verstärkten Selbst- bzw. Fremdbeobachtung verbunden ist, sondern auch bei dem Betroffenen behandlungsförderliche Einstellungen generiert, wie z.B. der Erkrankung

\section{Tab. 3 Übersicht psychoedukativer Ansätze}

\begin{tabular}{|c|c|c|}
\hline \multirow[t]{2}{*}{ Psychoedukative Ansätze } & \multicolumn{2}{|l|}{ Setting } \\
\hline & Einzel & Gruppen \\
\hline \multirow[t]{3}{*}{ manualisiert } & Basco \& Rush, 1996 (1) & Bauer \& McBride, 1996 (2) \\
\hline & Lam et al., 1999 (9) & Wagner \& Bräunig (16) \\
\hline & Miklowitz \& Goldstein, 1997 (7) * & \\
\hline \multirow[t]{3}{*}{ nicht manualisiert } & Cochran, $1984(4)$ & Palmer et al., 1995 (11) \\
\hline & Perry et al., 1999 (13) & Patelis-Siotis et al., 2001 (12) \\
\hline & Frank et al., 1997 (6) & Scott et al., 2001 (14) \\
\hline
\end{tabular}


nicht mehr hilflos ausgeliefert $\mathrm{zu}$ sein und deshalb aktiv zum Wohlbefinden beitragen zu können. Diese Steigerung des Selbstwirksamkeitserlebens im Rahmen einer in der watzlawickschen Terminologie als „symmetrisch“ bezeichneten Interaktionsbeziehung zwischen Arzt und Betroffenem, die beide $\mathrm{zu}$ gleichberechtigten Partnern in der therapeutischen Beziehung macht, dürfte Behandlungscompliance, Krankheitsverlauf und Krankheitsbewältigung sicherlich positiv beeinflussen. Entsprechende empirische Überprüfungen der potenziellen theoretischen Wirkprinzipien, insbesondere auch im Hinblick psychoedukativen Arbeitens mit Angehörigen, wären wünschenswert.

Obwohl die derzeit angewandten Untersuchungsdesigns zur empirischen Evaluation psychoedukativer Ansätze streng genommen noch nicht die Qualität von Efficacy- bzw. Effectiveness-Studien erreicht haben, leisten sie doch einen wichtigen Beitrag zur wissenschaftlichen Fundierung psychoedukativer Interventionen in der Behandlung bipolarer Erkrankungen.

\section{Literatur}

1. Basco MR, Rush AJ (Hrsg.). Cognitivebehavioral therapy for bipolar disorder. New York, The Guilford Press, 1996

2. Bauer M, Mc Bride L. Structured group psychotherapy for bipolar disorder. New York, Springer, 1996

3. Bäuml J, Pitschel-Walz G (Hrsg.). Psychoedukation bei schizophrenen Erkrankungen. Stuttgart: Schattauer, 2002

4. Cochran SD. Preventing medical noncompliance in the outpatient treatment of bipolar disorder. Journal of Consulting and Clinical Psychology 1984, 52: 873-878

5. Colom F, Vieta E, Martinez-Ará A, Reinares M, Goikolea J, Benabarre A, Torren C, Comes M, Corbella B, Parramon G, Corominas J. A randomized trial on the efficacy of group psychoeducation in the prophylaxis of recurrences in bipolar patients whose disease is in remission. Archives of General Psychiatry 2003; 60: 402-407

6. Frank E, Kupfer D], Ehlers CL, Monk TH, Corne C, Carter S, Frankel D. Interpersonal and social rhythm therapy for bipolar disorder: Integrating interpersonal and behavioral approaches. Behavior Therapist 1994; 17: $143-149$

7. Miklowitz DJ, Goldstein MJ (Hrsg.). A Family-Focused Treatment Approach. New York, Guilford Press, 1997

8. Miklowitz D, Simoneau T, George E, Richards J, Kalbag A, Sachs-Ericsson N, Sud- dath R. Family-focused treatment of bipolar disorder: 1-year effects of a psychoeducational program in conjunction with pharmacotherapy. Biological Psychiatry 2000; 48: 582-592

9. Lam DH, Jones SH, Hayward P, Bright JA. Cognitive therapy for bipolar disorder: A therapist 's guide to concepts, methods and practice. Chichester, John Wiley \& Sons, 1999.

10. 10.Lam DH, Watkins E, Hayward P, Bright J, Wright K, Kerr N, Parr-Davis G, Sham $\mathrm{P}$. A randomized controlled study of cognitive therapy for relapse prevention for bipolar affective disorder. Archives of General Psychiatry 2003, 60: 145-152

11. 11.Palmer A, Williams H, Adams M. Cognitive behaviour therapy in group format for bipolar affective disorder. Behavioural and Cognitive Psychotherapy 1995, 23: 153-168

12. 12.Patelis-Siotis I, Young LT, Robb JC, Marriott M, Bieling PJ, Cox LRT. Group cognitive behavioral therapy for bipolar disorder: A feasibility and effectiveness study. Journal of Affective Disorders 2001, Special Issue 65 (2): $145-153$

13. 13.Perry AN, Tarrier N, Morriss R, Mc Carthy K, Limb K. Randomised controlled trial of efficacy of teaching patients with bipolar disorder to identify early symptoms of relapse and obtain treatment. British Medical Journal 1999, 318 (7177): 149-153

14. 14.Scott J, Garland A, Moorhead S. A pilot study of cognitive therapy in bipolar disorders. Psychological Medicine 2001, 31: 459-467

15. 15.van Gent E, Zwart F. Psychoeducation of partners of bipolar-manic patients. Journal of Affective Disorders 1991, 21 : $15-18$

16. 16.Wagner $P$, Bräunig $P$ (Hrsg.). Psychoedukation bei bipolaren Störungen - Ein Therapiemanual für Gruppen (Druck in Vorbereitung)

17. 17. Yalom ID. The Theory and Practice of Group Psychotherapy. New York, Basic Books, 1975

\section{Key Words}

Bipolar affective disorder - psychoeducation - self-efficacy - encouraging empirical results

\section{Abstract}

Based on a biologically determined etiological concept, pharmacotherapy has long been the sole treatment strategy for this disorder. Empirical findings, however, showed that despite their compliance with pharmacotherapy, nearly $75 \%$ of subjects suffering from this disorder relapse within a 5-year-period., This led to the integration of psychotherapeutic interventions in the treatment of bipolar disorders. In addition to pharmacotherapy, psychoeducation seems to be a promising method for coping with the illness. Psychoeducation is a tool by which knowledge $a b-$ out the disorder and its treatment positively influences the course of the illness, enhances coping and improves patients' adherence to medication and thus reduces relapse rates.

\section{Korrespondenzadresse:}

Prof. Dr. med. habil. Peter Bräunig Klinikum Chemnitz gGmbH Klinik für Psychiatrie, Verhaltensmedizin und Psychosomatik

Dresdner Str. 178

09131 Chemnitz 